Témoigner Témoigner. Entre histoire et mémoire

Getuigen Revue pluridisciplinaire de la Fondation Auschwitz

$121 \mid 2015$

Violences radicales en scène

\title{
Intertextualité juive du pogrom
}

\section{Carole Ksiazenicer-Matheron}

\section{Q OpenEdition}

\section{Journals}

Édition électronique

URL : https://journals.openedition.org/temoigner/3415

DOI : 10.4000/temoigner.3415

ISSN : 2506-6390

Éditeur :

Éditions du Centre d'études et de documentation Mémoire d'Auschwitz, Éditions Kimé

Édition imprimée

Date de publication : 1 octobre 2015

Pagination : 141-151

ISSN : 2031-4183

\section{Référence électronique}

Carole Ksiazenicer-Matheron, «Intertextualité juive du pogrom », Témoigner. Entre histoire et mémoire

[En ligne], 121 | 2015, mis en ligne le 01 octobre 2016, consulté le 04 février 2022. URL : http:// journals.openedition.org/temoigner/3415; DOI : https://doi.org/10.4000/temoigner.3415 


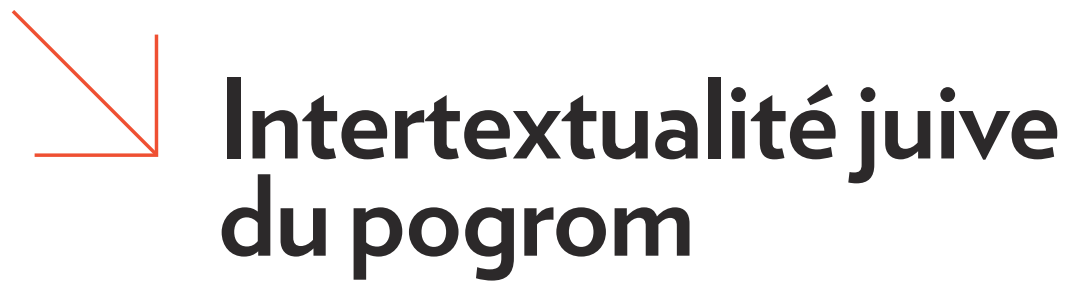

$\rightarrow$ Par Carole KsiazenicerMatheron, Université Sorbonne Nouvelle-Paris 3
Les textes évoqués ici représentent un aspect de l'écriture du pogrom, principalement en yiddish, et occasionnellement en hébreu ou en russe. Ils émanent d'auteurs qui pour la plupart, écrivent au $\mathrm{XX}^{\mathrm{e}}$ siècle, à la lumière des formes modernes de judéophobie qui sévissent en Europe orientale.

Les formes littéraires adoptées frappent par la multiplicité de leurs approches stylistiques : proches du document historique, ou bien fictionnelles, s'inscrivant dans le cadre du roman historique en yiddish, humoristiques, dans la lignée d'un Sholem-Aleykhem ou au contraire dramatisées par l'approche poétique et l'écriture moderniste. Par ces moyens de mise en lumière stylistique apparaissent à la fois l'horreur de l'histoire collective et les réponses que l'écriture, à la suite des rituels de deuil et de commémoration, autorise, dans le souci constant d'unir le texte moderne à la chaîne des énoncés pris en charge par la tradition mémorielle et littéraire.

Un bref rappel historique permettra de situer ces textes, dans le cadre des formes modernes d'antisémitisme qui se développent à la fin du XIX ${ }^{\mathrm{e}}$ siècle dans les confins occidentaux de l'empire russe, jusqu’à la guerre civile en Ukraine, qui anticipe déjà certaines formes massives d'extermination propres à la période génocidaire.

\section{CONTEXTE HISTORIQUE}

Avec l'assassinat du tsar Alexandre II au printemps 1881, la Russie tsariste, qui englobe une partie de la Pologne et l'Ukraine, connaît des vagues successives de pogroms sans commune mesure avec le système antérieur de discrimination. La politique partielle de libéralisation menée par le tsar assassiné trouve alors son terme définitif. La répression qui s’abat sur les mouvements révolutionnaires atteint par contrecoup, et selon la tactique du bouc-émissaire livré en pâture à l'anxiété des masses, toute la société juive, majoritairement coincée dans la zone de résidence, étranglée économiquement et touchée de plein fouet par une aggravation constante de son statut légal. La judéophobie officielle, relayée par une presse xénophobe et réactionnaire, alimentée par des rumeurs forgées de toutes pièces à propos de la « conspiration des Sages de Sion » et de la nocivité du Talmud, fait alors des ravages, tant dans ses formes policières et légales, que dans les débordements populaires qu'elle autorise, voire qu'elle suscite et couvre de son autorité. L’Église orthodoxe, 
les intellectuels slavophiles, les groupes paramilitaires comme les redoutables centuries noires, mais aussi une grande partie de la population locale applaudissent aux pogroms ou y participent activement.

Les premières exactions ont lieu en Russie du Sud et enclenchent le processus de la violence et des rumeurs. Le gouvernement, qui cherche à détourner l'attention de la situation intérieure, ne fait qu'attiser les troubles, et met bientôt en place un arsenal légal de « règlements provisoires » renforçant la discrimination. La dramatique expulsion des Juifs de Moscou en 1891 n'est qu'une des étapes de ce que l'historien Simon Doubnov a nommé les « pogroms légaux ». La jeunesse juive est littéralement barrée dans ses aspirations à accéder à l'instruction, la population misérable des bourgades est fixée dans ses lieux de résidence sans aucune perspective d'amélioration économique, l'intelligentsia est découragée dans sa volonté de libéralisation et de modernisation. Malgré la censure, les écrivains vont se tourner vers le yiddish pour réfléchir l'intense désespoir des masses et leur propre sentiment d'impasse quant à la rénovation du collectif, dans le cadre d'une politique de plus en plus répressive incitant au repli communautaire.

En 1903, le sauvage pogrom de Kichinev, qui fait une cinquantaine de victimes, de très nombreux blessés et accule à la ruine, matérielle et psychique, une grande partie de la population, anticipe l'aggravation de la situation, avec à sa suite la guerre russo-japonaise, la défaite russe et la tentative de Révolution de 1905.

Beaucoup plus sanglants et nombreux, les pogroms de cette période sont plus systématiquement organisés par la police secrète et voient une participation accrue de la population paysanne, surtout en Ukraine où le mouvement nationaliste se développe à partir de la reviviscence des mythes collectifs et de l'hostilité anti-juive. L'aire géographique des pogroms tend également à s'étendre, les tueries de Jitomir, de Bialystok, de Siedlce restant associées à la même image de barbarie que le pogrom de Kichinev. Le mouvement migratoire, en particulier vers les États-Unis, décisif dès les années 1880, s'intensifie, et dirige vers le Nouveau Monde une population plus politisée et plus éduquée. Le sionisme se développe également, alors que le climat intérieur se dégrade sous le gouvernement Stolypine, qui ressuscite les accusations médiévales de crime rituel, aboutissant au procès Beïlis, à Kiev. Ce dernier ne se clôt que peu de temps avant l'éclatement du conflit mondial, et de façon particulièrement ambiguë quant à l'accusation globale portée contre les Juifs, malgré l'acquittement de Beïlis.

En 1914, les espoirs que nourrit la société juive d'obtenir les droits civils en échange de sa participation loyale au conflit s'effondrent devant l'antisémitisme renforcé qui sévit au sein de l'armée, et jusque dans les sphères les plus proches de Nicolas II. La politique systématique de déportation de la population d'origine juive des zones de combats jette plus d'un demi-million de personnes sur les routes, les pogroms militaires perpétrés par les soldats russes accompagnant la retraite de 1915. Au début, les Juifs sont accusés d'être des espions, à cause de la proximité linguistique entre le yiddish et l'allemand, et sont souvent pris comme otages, exécutés, emprisonnés ou déportés en Sibérie sous le plus léger prétexte. 
Mais très vite, les exactions deviennent anarchiques et massives, en liaison avec le chaos et la désorganisation militaire. Plus que jamais, les Juifs, y compris les soldats au sein des régiments russes, deviennent de faciles boucs-émissaires, de simples proies à piller et à détruire.

Cette situation se retrouve, malgré des différences considérables, lors des événements de la guerre civile en Ukraine, à partir de 1917, où, aux pogroms militaires sporadiques liés au retour de l'armée, succèdent bientôt, à partir de 1919, les tueries systématiques dans le camp du chef nationaliste ukrainien Simon Petlioura, puis les pogroms de Denikine et des généraux blancs, ainsi que ceux des armées polonaises, et parfois même certains pogroms rouges. Les atamans, les traditionnels chefs cosaques, sévissent à la tête de leurs troupes, pour des

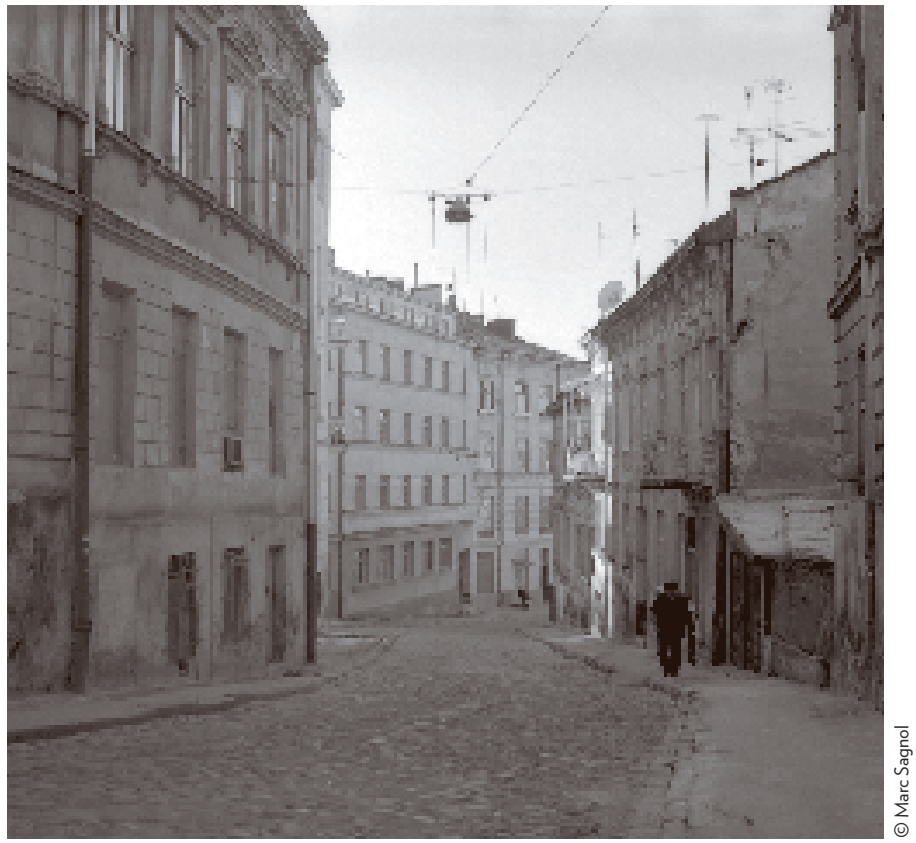
causes diverses, mais avec la même férocité.

Les bourgades juives sont prises en tenaille par la guerre civile, et les Juifs sont accusés à la fois d'être des accapareurs, des alliés de Moscou et des bolcheviques, soumis à une haine de classe féroce du côté de la population paysanne, et abandonnés par l'intelligentsia ukrainienne, acquise au nationalisme et au séparatisme. Le caractère apocalyptique des destructions, l'attaque délibérée contre la culture juive dans sa totalité, font déjà penser aux événements qui se dérouleront pendant la Seconde Guerre mondiale.

En Pologne, l'indépendance retrouvée à l'issue de la guerre se solde par une série de pogroms, à Lvov mais aussi à Cracovie, Kielce, Lublin, Wilno. L'humiliation des Juifs, dans les trains, les postes-frontières, les lieux publics est systématique. Mais c'est lors du conflit polono-bolchevique de 1920 que les destructions sont les plus importantes, sous couvert de lutte contre l'ennemi rouge. Après la guerre, le boycott économique commence à se mettre en place, en liaison avec le Kulturkampf et le progressif durcissement du régime, jusqu'à l'installation de l'idéologie ouvertement anti-juive des années trente, préfigurant la tragique disparition du judaïsme polonais pendant l'occupation nazie.

\section{TÉMOIGNAGE DE LA LITTÉRATURE}

La littérature témoigne de cette réalité implacable. Les textes s'alimentent encore aux énoncés traditionnels valorisant le martyre au nom de la foi, mais ils en subvertissent le sens sous l'effet de la révolte humaniste contre la vision religieuse.
_ Lvov (Galicie), rue Sanska, ancienne rue Boznica (2003). 
Ainsi, à la figure des victimes viennent s'adjoindre celle des résistants, s'opposant par les armes à l'oppression, ou celle du témoin, réfléchi par l'écrit, attestant par sa protestation solitaire le tragique destin collectif. La naissance de l'historiographie moderne relaie également la perpétuation de la mémoire ordinairement prise en charge par la sphère religieuse. L'écrivain et l'intellectuel juifs font encore appel aux archétypes, mais ils en transforment profondément le sens et la finalité, par le biais d'un éclatement des références et d'une volonté de perpétuer l'Histoire sous une forme scientifique, pour l'historiographie, ou artistique, pour la littérature. L'écriture réaliste elle-même tend à se dégager des formules typisées de la tradition mémorielle, même si elle les intègre encore de façon presque obsédante. L'humour, l'allégorie, le fantastique, la poésie s'emparent de la matière historique, pour la couler en des formes autonomes, propres à chaque tempérament stylistique, à chaque genre littéraire. Cependant, la fonction du témoignage persiste à travers cette palette variée de styles et de moyens d'énonciation. L'écrivain, hanté, halluciné par la vision historique, prête sa voix à la collectivité souffrante tout en plongeant au plus profond de sa psyché et de son art. Nous articulerons ce libre parcours des textes à la vision d'une évolution de l'écriture, qui part du réalisme documentaire pour aboutir aux formes déconstruites et iconoclastes de la modernité. À travers ces langues, ces styles, ces voix multiples, nous entendrons résonner un lot de souffrances qui mérite d'être rappelé, à l'aube d'une modernité qui avère son pouvoir infini de destruction mais aussi de résistance.

\section{LE MOUVEMENT DE L'HISTOIRE}

Nous commencerons par évoquer le texte bivocal de Simon Doubnov. C'est en russe qu'il écrit en 1916 son Histoire d'un soldat juif: histoire nous dit-il dont « le fond, sinon la forme est strictement historique » (Doubnov, 1988, 15), fiction d'histoire, d'après les lettres d'un soldat juif mort au front, à partir desquelles le grand historien du judaïsme développe le paradigme exemplaire d'une génération née avec les pogroms de 1881 et fauchée par le meurtrier conflit mondial et l'acharnement judéophobe du régime tsariste. Cette pseudo-autobiographie, forgée nous dit Doubnov « sous forme d'essai historique, à partir de faits connus, d'observations et de documents encore inédits » (Doubnov, 1988, 17) est «l'histoire personnifiée de la guerre contre les Juifs, d'une guerre qui dura 35 ans et qui sera exposée en détails, documents scientifiques à l'appui, dans un autre ouvrage » (Doubnov, 1988, 18) : il désigne ainsi son œuvre d'historien, l'Histoire universelle du peuple juif à laquelle il s'est attelé depuis 1903, date du pogrom de Kichinev. Le - je - qui raconte est fictivement celui du soldat, mais la voix qui s'exprime intègre le savoir de l'historien, pétrie d'empathie et de douleur. Le récit de la retraite de l'armée russe devant les troupes austro-allemandes, au cours de laquelle la fureur de l'armée en déroute se reporte contre les populations juives qu'elle est censée défendre, porte le sceau des matrices culturelles auxquelles se réfère l'historien, en une tentative constante de relier le présent au passé, l'histoire à la mémoire, la contingence au sacré. Présentée 
comme une force naturelle irrépressible, la violence militaire, dégénérant en pogroms sanglants, porte atteinte à la culture juive dans sa dimension humaine et symbolique, et débouche sur l'image puissante du martyrologe, en une audacieuse reprise du symbolisme chrétien de la crucifixion, réendossé par la collectivité juive dans sa totalité.

Dela méthodehistorique développée par Doubnov, l'écrivain yiddish Israël Joshua Singer a peut-être retenu l'aspect de linéarité et de totalité, dans sa fresque des Frères Ashkénazi qui a pour cadre Lodz, depuis la fin de la période napoléonienne jusqu’à l’indépendance de la Pologne. La scène de pogrom qui se situe à la fin du roman se déroule à Lvov, anciennement Lemberg, à la suite du conflit qui oppose les Polonais et les Ukrainiens pour le contrôle de la ville, en novembre 1918. Chez Singer, chaque étape

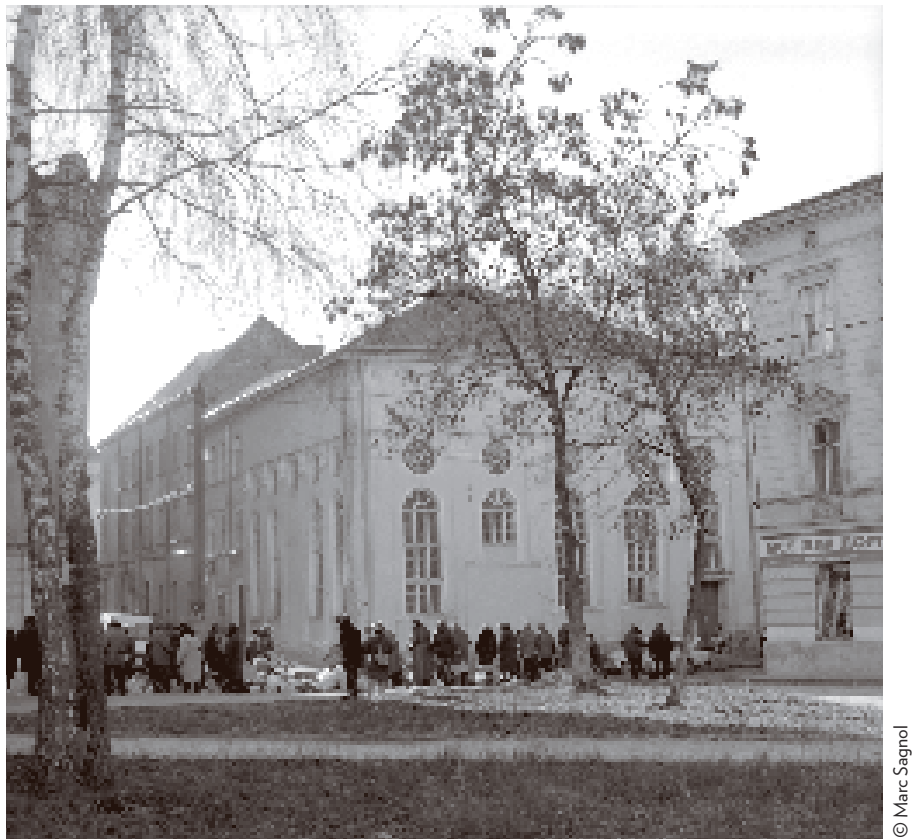
du développement historique se clôt dramatiquement par l'intersection violente de l'histoire des « nations » et de l'histoire juive, sous la forme paradigmatique du pogrom, que ce soit au moment du développement du capitalisme sauvage qui s'empare de la ville, ou lors des affrontements du pouvoir tsariste et du mouvement ouvrier, ou encore, comme ici, à la suite du conflit mondial, au moment de la constitution d'États-nations indépendants. La leçon du récit dessine un mouvement circulaire de néantisation du progrès et de retour mythique à l'origine, niant le processus d'assimilation des Juifs à la culture moderne. L'évocation a la précision glacée du document journalistique, du compte-rendu objectif. Le pogrom constitue un espace microcosmique, régi par une loi d'airain et une temporalité aussi systématique que celle du progrès mécanique, alliant la brutalité moderne à la cruauté haineuse de la foule. Le ratissage du quartier juif par l'armée, qui le transforme en souricière, le pillage organisé des biens juifs, la violence bestiale perpétrée par la population locale, l'incendie et la destruction des lieux et des objets de culte s'imposent au récit, en un enchaînement impitoyable, qui culmine avec la sortie hallucinée des rescapés du massacre, à la recherche des morts et des rouleaux de la Torah, enterrés conjointement. C'est déjà tout l'espace calciné de la vie juive en Pologne qui est évoqué dans la fin de ce roman, écrit au milieu des années 1930, au moment de l'émigration de l'auteur aux États-Unis. À l'inverse de Doubnov, la fresque singérienne, dans sa monumentalité même, ne laisse apparaître aucune voix subjective qui en ferait le récit, comme si la figure du témoin elle-même s'absentait, au cœur du processus d'aliénation et de déterritorialisation qui caractérise sa vision de l'histoire. 


\section{L'IMAGINAIRE DE L'HISTOIRE}

C'est une autre approche du roman historique en yiddish, marquée au contraire par l'imaginaire et la réécriture des chroniques traditionnelles, que dessine le roman d'Isaac Bashevis Singer, Satan à Goray, paru en Pologne en 1933, et celui de Sholem Asch, La Sanctification du Nom, paru en 1920. Tous deux s'inspirent des massacres de 1648 perpétrés par les cosaques ukrainiens de Bogdan Khmelnitski, relatés dans la chronique de Nathan Hannover, Le Fond de l'abîme, paru en hébreu en 1653.

Les deux textes modernes reprennent le motif de la persécution des « communautés saintes » qui forme l'armature du texte ancien : persécution de Zamość et des bourgades de la région de Lublin, chez Singer, persécution de Nemirov, chez Asch. La toute-puissance de l'armée cosaque est associée à une sauvagerie sadique, animalisée, exotique : image de l'altérité absolue. Mais alors que le texte de Singer, qui ouvre le roman, prend le parti d'une stylisation épique et sobre, très fidèle malgré ses coupes au récit initial, celui de Sholem Asch opte pour le réalisme pictural, travaillant sur l'atmosphère, les clairs-obscurs, l'alternance entre cris et silence, l'évocation d'un univers hybride lié à la profanation, avec une insistance ambiguë sur les éléments d'érotisation présents dans les scènes de rapt des femmes par les cosaques. Les deux textes synthétisent les aspects les plus suggestifs de l'horreur des massacres tels que les rapporte de façon plus détaillée, avec des procédés de liste et de typisation traditionnelle, Nathan Hannover : Singer avec le détail des femmes à qui l'on ouvre le ventre pour y coudre des chats, Asch avec celui des corps des enfants juifs débités comme de la viande casher vendue à la criée. Les deux descriptions culminent avec la notion de désacralisation de l'espace juif, intégrant la destruction des symboles cultuels. Dans les deux textes également, on relève un subtil contrepoint à la notion traditionnelle de Sanctification du Nom (Kiddush-hashem), le martyre au nom de la foi, valorisé par la chronique : chez Singer, la notion n'apparaît tout simplement pas et le texte se clôt sur l'effacement de la bourgade de la surface de la Terre, rappelant les visions apocalyptiques des villes bibliques livrées à la colère divine. Chez Sholem Asch, dont le roman reprend, par son titre, cette notion religieuse centrale, le texte est précédé d'un exergue, un passage des Psaumes soulignant la fidélité d'Israël à l'alliance, manière de mettre en relief une protestation implicite contre le destin du peuple élu. Singer ouvre le roman sur cette violence inhumaine, préhistoire de la crise messianique de 1666 liée au nom de Sabbataï Tsvi. Il s'attache à peindre avec ironie et grotesque le pandémonium qui règne à Goray, la bourgade juive prototypique. Quant à Sholem Asch, il choisit à l'inverse le merveilleux légendaire, la vision folklorisée de la survie miraculeuse de la nation « sainte », en dépit des atrocités de l'histoire.

\section{HUMOUR ET RÉSILIENCE}

Cette survie énigmatique du collectif est également au centre du texte d'Oser Warszawski, extrait des Contrebandiers, publié en Pologne en 1920. Texte écrit dans 
un yiddish argotique et populaire, non dénué de lyrisme, influencé conjointement par le naturalisme et l'expressionnisme. C'est parfois aussi la veine folklorique qui domine, avec la figure du petit tailleur, qui fait penser aux contes, à SholemAleykhem, et même à Sholem Asch, dont la Sanctification du Nom se termine justement sur cette figure légendarisée, image de la résistance culturelle. Même motif, chez Warszawski, mais l'humour et la dérision l'emportent sur le pathétique. Centré sur l'épisode de l'occupation allemande à Varsovie au début de la Première Guerre mondiale, le texte restitue sur le mode humoristique le climat de terreur dû aux accusations d'espionnage perpétrées contre les Juifs par les Polonais auprès du haut commandement russe. Par son habileté d'artisan et sa faconde courageuse et simple, un petit tailleur sauve de l'exécution les otages juifs de la bourgade. Un passage savoureux du récit met en scène de façon théâtralisée une oralité stylisée, mimétique du discours populaire, jouant du contraste entre terreur et ingéniosité langagière. Le motif de la suture invisible du vêtement vaut pour manifeste esthétique, à l'image d'un texte travaillé par la réminiscence des personnages et du style de Sholem-Aleykhem. C'est toute la collectivité juive menacée de disparition qui s'exprime à travers la référence à un langage assurant à lui seul le miracle de la survie, contre la violence des pouvoirs officiels. Fraudant avec la loi, subvertissant l'ordre mortifère qui se met en place à la faveur de la guerre, les contrebandiers font passer malgré tout leur message chimérique d'espoir et de vitalité, alimenté par les ressources de la langue, stylisé par le talent mimétique de l'écrivain.

\section{DANS LA VILLE DU MASSACRE}

Avec le poème de Bialik La Ville du massacre, réécrit par le poète en yiddish après une première version en hébreu, c'est d'une autre postérité qu'il s'agit : celle qui inspire une poésie moderniste blasphématoire, dont fait également partie le long cycle poétique de Peretz Markish, Le Tas. Les deux poèmes entrent indéniablement en résonance. Bialik, le créateur de la poésie hébraïque moderne, l’intellectuel d'Odessa qui émigrera ultérieurement en Palestine, écrit immédiatement après le pogrom de Kichinev, où il a été envoyé pour enquêter sur les événements. Peretz Markish, le chantre en yiddish de la Révolution, liquidé par Staline en 1952, choisit comme paradigme de la terreur Horoditch, une bourgade d'Ukraine parmi tant d'autres martyrisées par la guerre civile. La date du pogrom fait référence non à l'histoire, mais à la sacralité du calendrier juif et à la fête de Kippour, souillée par le crime dont elle fait ressortir le caractère sacrilège. Les deux poèmes dans leur entier sont d'une longueur considérable, faisant se succéder les images de mort, rythmées par les invocations, les blasphèmes, les tableaux obscènes, la désacralisation concertée des archétypes religieux. Le statut de l'énonciation est dans chaque cas ambigu : le - je - poétique pourrait faire spontanément penser à la voix du poète, mais chez Bialik, c'est en réalité, par un procédé d'inversion ironique, Dieu lui-même qui invite l'homme-témoin à se révolter contre sa propre Loi, tandis que chez Markish, c'est le tas monstrueux des cadavres amoncelés qui interpelle un ciel vide, souillé par le « suif » sacrilège, 
la chair humaine violentée, et qui finira par vomir les dix Commandements à la fin du poème. Le registre prophétique cohabite avec la trivialité grotesque, la beauté poétique avec l'horreur spectaculaire, le discours de revendication avec le besoin de convoquer indéfiniment les références à un sacré désormais aboli. L'invitation à voir, à éprouver, à sentir, à toucher l'horreur du pogrom dessine ultimement un paysage surréel, où l'espace morbide devient la seule réalité, la seule éternité, avec celle de la voix poétique qui résonne au présent.

\section{MODERNISME ET DESTRUCTION}

La poésie en yiddish a contribué à briser le carcan de la langue, à créer une modernité ouverte à toutes les transgressions, à débarrasser la littérature des stéréotypes routiniers, du réalisme conventionnel, des missions pieuses de l'idéologie, qu'elle soit communautaire ou révolutionnaire. À sa suite, naissent des proses d'une liberté étonnante, les nouvelles de facture futuro-expressionniste d'Israël Joshua Singer, dont le récit En terre étrangère est consacré à la guerre civile en Russie; comme également les plus célèbres des nouvelles dédiées au pogrom en yiddish, celles de Lamed Shapiro dans son recueil de 1919 Le Royaume juif. Le registre épique lié à la guerre est sensible dans le texte singérien, de même que dans l'extrait de la Halla blanche de Shapiro, qui retrace un contexte finalement assez proche de celui de Doubnov dans L'Histoire d'un soldatjuif, mais avec une différence de moyens poétiques qui en fait toute la nouveauté. L’angoisse apocalyptique de la guerre, la terreur du pogrom, la menace quasi cosmique de destruction s'incarnent désormais dans une prose symboliste, dont les personnages ne sont plus les acteurs humains, mais des entités énigmatiques, détentrices de la violence selon des lois aussi cruelles qu'incompréhensibles. Le pogrom dans ces textes est devenu le chiffre de l'égarement humain, parmi des signes opaques désignant un ordre transcendant mais insensé. L’angoisse, la douleur, l'attente, la folie sont convoquées en tant qu'expériences ordinaires dans la vie des individus, écrasés par la peur et le non-sens. D’objet historique, le pogrom devient poétique, voire métaphysique. Le titre de la nouvelle En terre étrangère désigne l'exil en quoi consiste l'Histoire, non seulement l'histoire des Juifs mais l'histoire en tant que conscience moderne, subjective, telle qu'elle est exprimée par la littérature. C'est pourtant aux archétypes traditionnels que recourent ces auteurs exilés de la collectivité, comme si les signifiants, de par leur seule force langagière, gardaient encore une fonction de conservation de l'identité fragmentée par la violence. La nouvelle de Singer se termine sur les mots du kaddish prononcés sur la fosse commune où reposent les soldats juifs du bataillon international ; la nouvelle éponyme du recueil de Shapiro intègre les réponses opposées à la violence par la communauté religieuse: les prières et l'écriture des chroniques retraçant les persécutions depuis des temps immémoriaux. Mais à la fin du passage dépeignant la communauté martyre regroupée dans l'espace protecteur de la synagogue fait irruption le fils du rabbin, qui, de l'intérieur même de l'espace juif, va détruire ces restes de sacralité par ses discours blasphématoires, en des termes qui sont ceux du poème de Bialik. 
Le Dieu juif, la sainteté juive, l'éthique traditionnelles, toutes les valeurs qui donnaient sens à la vie collective sont anéanties : « des vies entières, des vies entières fracassées à jamais comme des crânes. » (Bialik, 1971, 62)

\section{FANTASMAGORIES DE LA VIOLENCE}

Né en Ukraine et émigré aux ÉtatsUnis au moment des pogroms de 1905, qu'il évoque avec la distance d'un style encore inédit dans la littérature yiddish, mais aussi avec l'empathie obsessionnelle (et peut-être la secrète culpabilité) de celui qui est loin des siens, Shapiro assigne à ses nouvelles un caractère délibérément fictionnel, les marquant de ses propres fantasmagories, transformant la scène historique en théâtre intime, hanté par une violence inouïe. La langue yiddish expérimente dans ses textes des distorsions vertigineuses. $\grave{A}$ peu près au même moment, c'est en russe qu'Isaac Babel, né lui aussi en Ukraine, à Odessa, évoque la cruauté de la guerre et de la Révolution, portant sur les souffrances juives un regard fortement ambivalent. Les deux nouvelles de 1925 Histoire de mon pigeonnier et Premier amour, évoquent par leurs titres l'univers de l'enfance que Babel, après Tourgueniev et Gorki, ressuscite

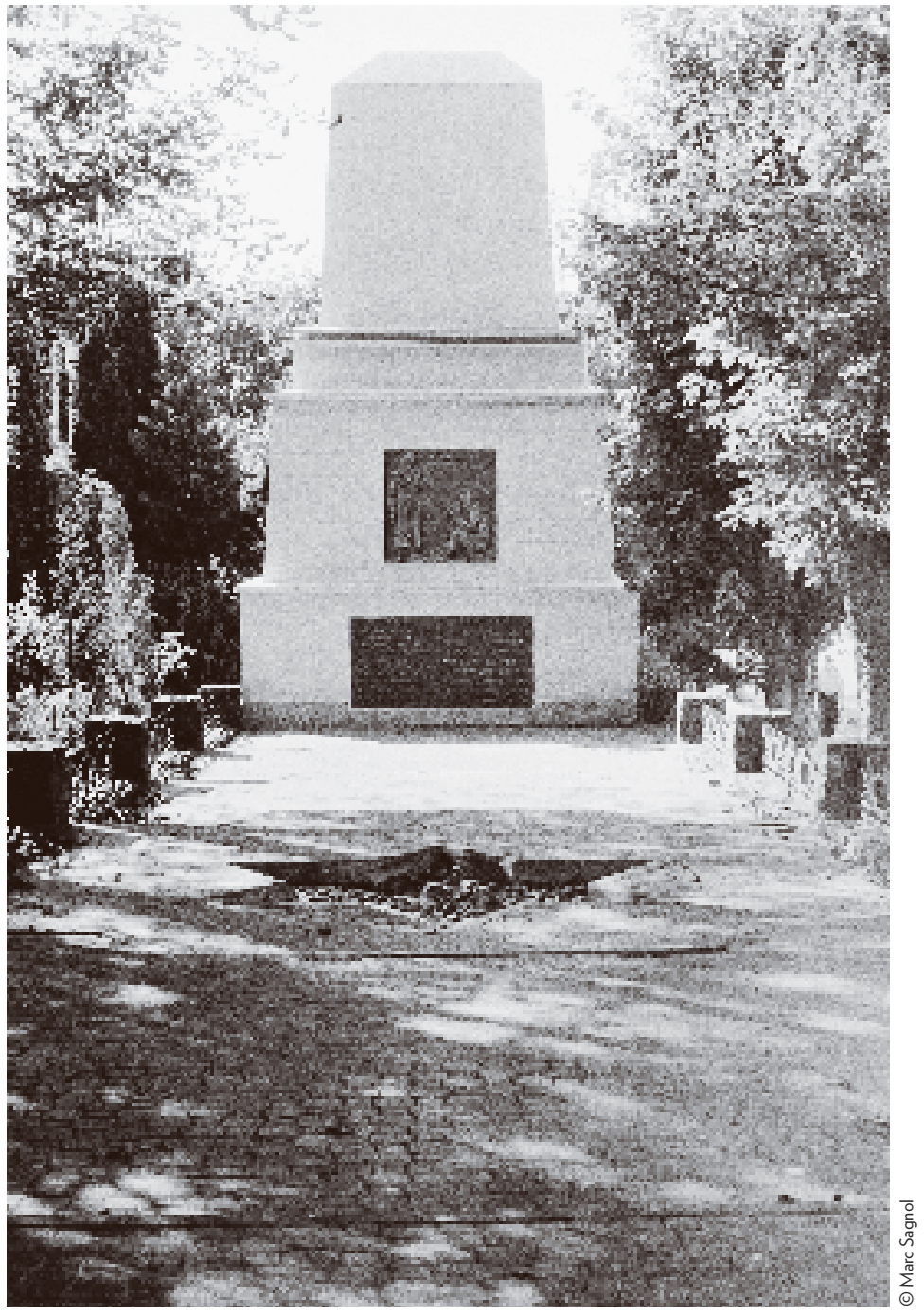

_ Monument aux victimes du pogrom de Proskourov (1919), Khmelnitsky (2003).

\section{intenses et de chagrins inoubliables, l'Histoire fait effraction, inspirant l'écriture} désir enfantin, d'acharnement scolaire et de récompense pour l'enfant juif qui a su sortir victorieux des embûches du numérus clausus, tel David triomphant de Goliath, le récit inclut en son centre l'histoire du pogrom de Nicolaev en 1905, auquel Babel, âgé d'une dizaine d'années a assisté. Comme chez Shapiro, le paradigme fictionnalisé du pogrom noue de façon exemplaire désir et culpabilité, transgression et violence: « Tout se passa selon mes désirs, tout se passa mal » (Babel, 1967, 137) mentionne 
le narrateur rétrospectif à la jonction des deux histoires, qui finalement n'en font qu'une : celle de l'achat des pigeons, ardemment convoités, et celle de l'éclatement du pogrom, qui transforme à jamais la vision du monde de l'enfant. Ici encore il est question d'intériorité fracassée, de viol de l'esprit et du corps, d'intimité détruite. La prostration enfantine après l'acte d'agression, l'errance quasi psychotique dans un paysage urbain surréaliste, l'inversion des affects et des signes sont analogues à un parcours initiatique, à une mort suivie d'une étrange renaissance : celle d'un enfantmessie orné de plumes, stigmates de la violence, s'ouvrant à l'unité ambivalente du monde, à travers l’alliance de la beauté et de la mort que transcrit le langage poétique.

\section{AMBIVALENCE ET ENGAGEMENT}

L'ambivalence poétique sur laquelle joue la fiction, y compris dans les récits de Cavalerie rouge qui retracent l'expérience de la guerre polono-bolchevique, est en partie liée à l'expérimentation esthétique. Elle est beaucoup moins ambiguë dans le Journal de 1920, non destiné à la publication, où Babel restitue son état d'esprit lorsqu'il accompagne le régiment bolchevique de Boudionny en Galicie et en Wolhynie. Même si Babel opte pour la Révolution, avec la nette conscience que la violence est intrinsèquement liée au processus de prise du pouvoir, il n'en est pas moins horrifié par le sort particulier fait à la population juive. Derrière la phraséologie révolutionnaire, à laquelle lui-même sacrifie puisqu’il rédige les bulletins de propagande pour l'agence de presse Rosta, il débusque la nature atavique de l'acharnement anti-juif dans l'armée cosaque, rappelant justement les souvenirs du temps de Khmelnitski :

« La haine est la même, les cosaques sont les mêmes de part et d'autre, avec la même cruauté, des armées différentes quelle bêtise ! La vie des shtetls. Aucun salut. Tout le monde les tue - les Polonais ne leur donnaient aucun abri. Les jeunes filles et les femmes peuvent à peine marcher. » (Babel, 1991, 178)

Dans ce court extrait du Journal, Babel assume un rôle de témoin, d'enquêteur même, un peu à la façon dont Ansky en 1915, plus ou moins dans les mêmes lieux, recueillait les témoignages des victimes des troupes russes et leur prodiguait des secours. Babel est loin, certes, de l'engagement d'un Ansky aux côtés des seules victimes juives. Cependant, la perception de la «peur et du désespoir indicibles » (Babel, 1991, 176) des Juifs qui se terrent dans des caves après un récent pogrom, au milieu du sang et parmi les morts, donne à ces notations brèves une économie nerveuse et sèche, une lucidité presque désespérée. Comme souvent, Babel transcende le compte-rendu objectif, presque sténographique, par l'emprunt spontané aux références juives : David Zis, le chef de famille, ressemble à un prophète nu ; quant à la liste des noms des victimes, elle évoque, dans son caractère elliptique, les inscriptions des chroniques traditionnelles, les pinkessim. Certes, le pogrom a été perpétré par les Polonais, mais Babel constate avec amertume : «L'effroi, la 
terreur de la population. Le pire, c'est que les nôtres passent, indifférents, et pillent où ils peuvent, dépouillent les morts.» (Babel,1991,177) Concluant sur une notation typique de son regard à la fois mélancolique et acéré sur la réalité historique qui se donne à lire à lui comme les couches superposées d'un palimpseste : «Quelle vie nationale puissante et charmante il y avait eu ici. » (Babel, 1991, 178) Distance non moins grande que celle qui sépare Shapiro, exilé aux États-Unis, de sa terre natale : distance temporelle et non plus spatiale, celle qui convoque un monde où l'image du passé subsiste en transparence, mais pour peu de temps, et seulement au regard attentif du narrateur et peut-être du lecteur.

\section{BIBLIOGRAPHIE}

- Ansky, 1921-1922, Der yiddisher khurbn fun Poyln, Galitsie un Bukovine, Gezamelte Shriften t. 4 et 5, Varsovie, Farlag Anski.

- Sholem Asch, 1985, La Sanctification du Nom, trad. du yiddish par Aby Wieviorka et Henri Raczymow, préface d'Itzhok Niborski, Lausanne, L'Âge d'Homme.

- Isaac Babel, 1967, Contes d'Odessa, trad. du russe par A. Bloch et M. Minoustchine, Paris, Gallimard, coll. «Folio ».

- Isaac Babel, 1991, Journal de 1920, trad. du russe par Wladimir Berelowitch, Paris, Balland.

- Khayim Nakhman Bialik, 1987, La ville du massacre, in Le Miroir d'un peuple, (Anthologie de la poésie yiddish), trad. par Charles Dobzynski, Paris, Seuil.

- Simon Doubnov, 1988, Histoire d'un soldat juif (1881-1915), trad. du russe par Laurence Dyevre et Alexandre Eidelman, préface de Léon Poliakov, Paris, Cerf.

- Nathan Hannover, 1991, Le Fond de l'abîme. Les Juifs dans la tourmente des guerres cosaco-polonaises (16481650), trad. de l'hébreu par Jean-Pierre Osier, Paris, Cerf.

- Peretz Markish, 2001, Le Tas, trad. du yiddish par Batia Baum, in Caravanes, Paris, Phébus.

- Lamed Shapiro, 1987, Le Royaume juif, trad. du yiddish par Delphine Bechtel, Carole Ksiazenicer et Jacques Mandelbaum, Paris, Seuil.

- Isaac Bashevis Singer, 2010, Satan à Goray, trad. de l'anglais par Marie-Pierre Bay avec la collaboration de Nicolas Bay, préface de Jacob Sloan, Paris, Stock.

- Israël Joshua Singer, 1989, En Terre étrangère, trad. du yiddish par Françoise Kreissler, in Une maisonnette au bord de la Vistule, Paris, Albin Michel.

- Israël Joshua Singer, 1993, Les Frères Ashkenazi, trad. de l'anglais par Marie-Brunette Spire, Paris, Denoël.

- Oser Warszawski, 1989, Les Contrebandiers, trad. du yiddish par Aby Wieviorka et Henri Raczymow, Paris, Seuil. 